\title{
Validity and Reliability of Korean Version of Behavior Problems Inventory in Autism Spectrum Disorder and/or Intellectual Developmental Disorder
}

\author{
Samuel Suk-Hyun Hwang', Jangho Park², Sohee Kim², \\ Gayoung Lee ${ }^{3}$, Yeni $\mathrm{Kim}^{4}$, and Soo-Young Bhang ${ }^{5}$ \\ 1 Department of Psychology, Chonnam National University, Gwangju, Korea \\ ${ }^{2}$ Ulsan University Hospital, College of Medicine, University of Ulsan, Ulsan, Korea \\ ${ }^{3}$ Graduate School of Professional Therapeutic Technology, Seoul Women's University, Seoul, Korea \\ ${ }^{4}$ Department of Child and Adolescent Psychiatry, National Center for Mental Health, Seoul, Korea \\ ${ }^{5}$ Department of Psychiatry, Eulji General Hospital, Eulji University School of Medicine, Seoul, Korea
}

\begin{abstract}
Objectives: Problem behaviors pose a great obstacle to daily functioning in children with neurodevelopmental deficits and are an important target for treatment. In this study, we translated the Behavior Problems Inventory (BPI)-01 into Korean language and tested its psychometric properties.

Methods: We attained the approval of the BPI author and performed standard translation-back translation. Then, professional caregivers examined appropriateness according to cultural and situational contexts in order to make necessary modifications of the Korean version of the BPI.

Results: The BPI-01, Korea-Scales of Independent Behavior-Revised (K-SIB-R), and Child Behavior Checklist were completed for 98 autistic spectrum disorder or intellectual development disorder participants (mean age=28.3, standard deviation=7.3, range $=10.1-51.7$ ). The inter-rater reliability of the BPI-01 was found to be high ( $\mathrm{r}=0.992-1.000)$. As for the results for concurrent validity for subscale items of BPI-01 and scales of SIB, correlations between SIB and BPI-01 subscales were statistically significant $(\mathrm{r}=0.357-0.672)$.

Discussion: The Korean version of the BPI-01 showed good psychometric properties with high reliability and sufficient convergent validity. Further examination of the validity of BPI-01 should be carried out with inclusion of younger aged children and a closer look at less frequently occurring symptoms.
\end{abstract}

Key Words: Autism spectrum disorder; Problem behavior; Stereotype; Behavior Problems Inventory.

Received: May 25, 2017 / Revision: August 12, 2017 / Accepted: August 22, 2017

Address for correspondence: Soo-Young Bhang, Department of Psychiatry, Eulji General Hospital, Eulji University School of Medicine, 68 Hangeulbiseokro, Nowon-gu, Seoul 01830, Korea

Tel: +82-2-970-8303, Fax: +82-2-970-8429, E-mail: bsy1@eulji.ac.kr

\section{INTRODUCTION}

Mental disorders in children such as intellectual developmental disorder (IDD) and autistic spectrum disorder (ASD) involve persistent behavioral problems. While determination of the prevalence of IDD is complicated due to different operational definitions and subjectivity in diagnosing IDD between clinicians, a recent meta-analysis of international studies found the prevalence of IDD in children and adolescents to range from $0.22 \%$ to $1.55 \%$. $^{1)}$ On the other hand, ASD appears to be somewhat less prevalent, lying within the range of 0.7 to 94 cases per 10000 people. ${ }^{2}$ The prevalence

This is an Open Access article distributed under the terms of the Creative Commons Attribution Non-Commercial License (http://creativecommons.org/licenses/by-nc/4.0) which permits unrestricted non-commercial use, distribution, and reproduction in any medium, provided the original work is properly cited. rate of ASD in Korea has been reported to be $2.64 \%$ [95\% confidence interval $(\mathrm{CI})=1.91-3.37] .^{3)}$ Such figures show that IDD and ASD are likely to occur as frequently as other major psychiatric disorders in Korea. The clinical manifestations of these disorders are diverse and underlie many problematic behaviors. According to the Diagnostic and Statistical Manual of Mental Disorders, 5th edition, ${ }^{4)}$ in addition to deficits in intellectual functions, such as language development and reasoning, IDD also shows adaptive functioning deficits in social domains including emotional and behavioral dysregulation that may negatively affect personal independence and social interactions. The clinical features of ASD include deficit in social communication and repetitive stereotyped interests and behaviors which are expressed verbally and nonverbally. ${ }^{4,5}$ Specifically, social communication 
deficits include lack of emotional exchange and use of nonverbal communication with others, as well as inability to form and maintain relationships with people other than parents that are appropriate for the developmental age. Stereotypical tendencies in behavior, interest, and activities include stereotyped repetitive movements, immediate and delayed echolalia, inflexible adherence to routines, and resistance to change, restricted and fixed interest, and sensitivity toward routine stimuli. Overall, maladaptive behavioral problems due to self-affliction, aggression toward others, overactive behavior, and stereotypical tendencies are common in neurodevelopmental disorders and make adjustment to society or facilities very difficult. ${ }^{6-8)}$

Availability of a reliable instrument to assess behavioral problems in IDD and ASD is extremely important for planning treatment strategy and evaluating treatment response. For example, increasingly more ASD patients are receiving drug treatment and the proportion of those on pharmacotherapy increases with age. However, rather than improving social interactions or communication problems, ${ }^{9)}$ the such pharmacotherapy targets comorbid symptoms such as anxiety, depression, compulsiveness, hyperactivity, attention deficit, sleep disturbance, and catatonic-like behavior or problems associated with aggression, self-harm, or severe stereotypical behaviors. ${ }^{5}$ Hence, an effective instrument should cover a wide range behaviors, such as stereotyped repetitive behavior, mannerism, and obsessions with dysfunctional habit or compulsive characteristics, and also examine aggression, impulsiveness, and self-harming behaviors.

Furthermore, in order to maximize the utility in clinical setting, the rating scale should be easy to use and not too lengthy as semi-structured interviews require relatively more time and trained rater. While including sufficient number of items in the rating scale to cover the wide breadth of problematic behaviors is important, but having too many items which are not pertinent to problematic behaviors characteristic of the specific disorders can pose excessive burden on the raters. For example, a widely used Korean-Scales of Independent Behavior-Revised (K-SIB-R), ${ }^{10)}$ which is sectioned into independent functioning behaviors and problematic behaviors contain 259 items. On the other hand, the KoreaChild Behavior Checklist (K-CBCL) $)^{11)}$ contains problematic behavior syndrome scale consisting of 119 items. Though it contains fewer items, the contents of each subscales of the CBCL appears inadequate to address the characteristic problematic behaviors of IDD and ASD children, since the items generally relate to non-clinical children.

In this study, we examined the psychometric properties of the Behavior Problems Inventory-01 (BPI-01) which has been translated into more than 10 languages. ${ }^{12)}$ It focuses primarily on the comprehensive range of behavioral problems and includes a total of 52 items, which are assessed in two dimensions of frequency and severity. Through the standard translation process and a careful examination of the reliability and validity of the scale, we evaluated the adequacy of the BPI- 01 as a scale which can be applied to the assessment of symptoms and treatment response ${ }^{13)}$ in ASD patients in Korea.

\section{METHODS}

\section{Data collection}

This protocol of this study was approved by the ethics committee of the Eulji University Hospital (IRB No. 2013-10-008). The permission to translate BPI-01 into Korean for validation was obtained from its original author. ${ }^{14)}$ After standard translation process, the translated BPI- 01 was administered to people residing in a residential facility for examination of its validity and reliability. After standard translation process, the translated BPI-01 was administered to people residing in a residential facility with informed consent for examination of its validity and reliability.

\section{Translation of the BPI-01}

The BPI- 01 was translated into Korean by SYB and then back-translated by SSH to check for appropriateness of the Korean translation. The Korean version of the BPI-01 was examined by the on-site profession caregivers for clarity and appropriateness of the items. The final Korean version of the BPI-01 was derived after a Korean language specialist checked for linguistic adequacy.

\section{Participants}

A total of 98 participants with a mean age of 28.3 years [standard deviation (SD)=7.3, 10.1-51.7 years] were includ-

Table 1. Demographic characteristics of the participants $(n=98)$

\begin{tabular}{lc}
\hline & Number $(\%)$ \\
\hline Male & $71(72.4)$ \\
Female & $27(27.6)$ \\
Age (mean \pm SD) & $28.3 \pm 7.3$ \\
Diagnosis & \\
IDD & $39(39.8)$ \\
ASD & $19(19.4)$ \\
IDD+ASD & $34(34.7)$ \\
Unknown & $6(6.1)$ \\
Medication & $71(72.4)$ \\
Yes & $23(23.5)$ \\
No & $4(4.1)$ \\
Unknown & \\
\hline
\end{tabular}

ASD: autistic spectrum disorder, IDD: intellectual developmental disorder, SD: standard deviation 
ed in our study. The responses were obtained from professional caregivers who spent at least 6 hours per day with the participants. The mean duration of supervision by these caregivers was 14.2 months ( $\mathrm{SD}=12.1$ months). The diagnoses of these participants were mostly moderate level of IDD or ASD. Among them, 71 (71.6\%) were receiving psychiatric medication. The demographic data of our participants are presented in Table 1.

\section{Instruments}

\section{BPI-01}

The original BPI version was initially developed in Germany to be used for assessing severe problematic problems. ${ }^{12)}$ Its application can be extensive, from rating problematic behaviors and assessing treatment outcomes in clinical settings and analyzing behavioral data in epidemiological studies to making administrative decisions such as determination of admission into residential treatment centers. The original version consisted of items measuring self-harming and stereotyped behaviors and in the process of translating into English, the researchers added aggressive/destructive items. Finally, 5 items pertaining to stereotyped behaviors were replaced by the "stereotyped behavior subscale" and the present name BPI-01 was given to the scale. It includes a total of 52 items and the problem behaviors are assessed in two dimensions of frequency (never $=0$, monthly $=1$, weekly $=2$, daily $=3$, hour$l y=4)$ and severity (mild $=1$, moderate $=2$, severe $=3$ ). There are 14 items in the self-harming behavior, 24 items in stereotyped behavior, and 11 items in aggressive/destructive behavior. The respondents were asked to rate the behaviors which occurred at least once during the previous two months and they could also list one additional behavior item that is not included in the scale.

\section{K-SIB-R}

To examine the concurrent validity of the BPI-01, we used the problem behavior subscale of the K-SIB-R. ${ }^{10)}$ The K-SIB-R is divided into two sections, i.e., independent functioning behaviors, which include motor skills, social interactions and communications, personal life skills, community life skills, and problematic behaviors which assess internal maladaptive behaviors (self-harming behavior, odd stereotyped habits, withdrawal or inattentive behavior), external maladaptive behaviors (other-harming behavior, destructive behavior, obstructive behavior), antisocial maladaptive behaviors (socially aggressive behavior, uncooperative behavior).

\section{K-CBCL}

For examination of discriminant validity, K-CBCL, ${ }^{11)}$ origi- nally developed by American psychologists Achenbach and Edelbrock $^{15)}$ and then translated into Korean was applied to this study. The problematic behavior syndrome scale consists of 119 items scored on a 3-point scale with low scores indicating mild levels of problematic behaviors. As the characteristics of problem behaviors and the contents of each subscales of the K-CBCL generally pertain to those of non-clinical children, we determined that the nature of the problematic behaviors reflected by the BPI- 01 should be qualitatively distinguishable from that of the K-CBCL.

\section{Statistical analysis}

For internal reliability, we obtained the Cronbach's a coefficient and item-total correlations of all items in the scale. We also examined inter-rater correlations and 2-week testretest reliability using Spearman's correlation coefficient. All other correlation analysis between BPI-01 and K-SIB-R and K-CBCL were carried out also using Spearman's correlation coefficient. All analysis were carried out using SPSS version 18 (SPSS Inc., Chicago, IL, USA).

\section{RESULTS}

\section{BPI-01 translation}

While the previous Korean version of the BPI-01 has been published, ${ }^{16)}$ some key items were modified in our study for a more accurate translation and to better capture the subtle nuances of the original version. For example, item 11 ('air swallowing resulting in extended abdomen') previously translated as 'swallowing much air to make the stomach bulge out' was modified as 'swallowing air resulting in bulging stomach.' Likewise, a number of items in the stereotyped behavior subscale underwent similar modifications: item 8 ('pacing') of the stereotyped behavior subscale previously translated as 'continuing to walk in short steps' was modified as 'repetitively going back and forth in the same place': item 10 ('having repetitive hand movements') was revised from 'moving hands repetitively' to 'having repetitive movement of hand (s)': item 15 ('having bursts of running around') was revised from 'going around ceaselessly' to 'sporadic sudden running': item 16 ('engaging in complex hand and finger movements') from 'moving hand or fingers in a complex way' to 'showing complex hand or finger movements,' and item 18 ('exhibiting sustained finger movements') from 'moving fingers continuously' to 'continuous (or continuously sustained) finger movements.' Lastly, a stereotyped behavior of 'head turning from side to side' was frequently observed in our assessment hence it was added to the stereotyped subscale to be rated. In terms of severity scale, the original study used a scale ranging from 0 to 3, but we applied a 5-point scale for a better distinction: 
Table 2. Item statistics: psychometric properties of the Behavior Problems Inventory-01 severity data

\begin{tabular}{|c|c|c|}
\hline Subscales and items & Item-total & Test-retest \\
\hline \multicolumn{3}{|l|}{ Self-injury behavior subscale } \\
\hline 1. Self-biting & $0.570^{* *}$ & $0.771^{* *}$ \\
\hline 2. Head-hitting & $0.571^{* *}$ & $0.629 *$ \\
\hline 3. Body-hitting & $0.441^{* *}$ & $0.879 * *$ \\
\hline 4. Self-scratching & $0.476^{* *}$ & -0.118 \\
\hline 5. Vomiting & 0.159 & $0.647^{* *}$ \\
\hline 6. Self-pinching & $0.261^{*}$ & $1.000^{* *}$ \\
\hline 7. Pica & $0.457^{* *}$ & $0.530^{*}$ \\
\hline 8. Stuffing objects & 0.186 & - \\
\hline 9. Nail-pulling & $0.450^{* *}$ & $0.686^{* *}$ \\
\hline 10. Pocking & $0.464^{* *}$ & - \\
\hline 11. Aerophagia & 0.096 & - \\
\hline 12. Hair pulling & $0.584^{* *}$ & $1.000^{* *}$ \\
\hline 13. Drinking & $0.267^{* *}$ & - \\
\hline 14. Teeth-grinding & $0.221^{*}$ & 0.395 \\
\hline 15. Other & $0.328^{* *}$ & - \\
\hline \multicolumn{3}{|l|}{ Stereotyped behavior subscale } \\
\hline 1. Rocking back and forth & $0.624^{* *}$ & $0.560^{*}$ \\
\hline 2. Sniffing objects & $0.514^{* *}$ & -0.102 \\
\hline 3. Spinning own body & $0.563^{* *}$ & $0.681^{* *}$ \\
\hline 4. Waving or shaking arms & $0.597^{* *}$ & 0.475 \\
\hline 5. Rolling head & $0.430 * *$ & - \\
\hline 6. Whirling, turning around on spot & $0.542^{* *}$ & 0.097 \\
\hline 7. Engaging in repetitive body movements & $0.731^{* *}$ & 0.196 \\
\hline 8. Pacing & $0.607^{* *}$ & $0.590^{*}$ \\
\hline 9. Twirling things & $0.273^{* *}$ & - \\
\hline 10. having repetitive hand movements & $0.532^{* *}$ & 0.244 \\
\hline 11. Yelling and screaming & $0.351^{* *}$ & $0.878^{* *}$ \\
\hline 12. Sniffing own body & $0.433^{* *}$ & - \\
\hline 13. Bouncing around & $0.546^{* *}$ & 0.402 \\
\hline 14. Spinning objects & $0.400 * *$ & $1.000^{* *}$ \\
\hline 15. Having bursts of running around & $0.450^{* *}$ & 0.438 \\
\hline 16. Engaging in complex hand and finger movement & $0.472^{* *}$ & $0.969^{* *}$ \\
\hline 17. Manipulating objects repeatedly & $0.550 * *$ & -0.105 \\
\hline 18. Exhibiting sustained finger movements & $0.304^{* *}$ & - \\
\hline 19. Rubbing self & $0.398^{* *}$ & - \\
\hline 20. Gazing at hands or objects & $0.419^{* *}$ & 0.419 \\
\hline 21. Maintaining bizarre body postures & 0.197 & 0.425 \\
\hline 22. Clapping hands & $0.360^{* *}$ & 0.654 \\
\hline 23. Grimacing & $0.542^{* *}$ & 0.433 \\
\hline 24. Waving hands & $0.487^{* *}$ & - \\
\hline 25. Other & $0.349^{* *}$ & - \\
\hline \multicolumn{3}{|l|}{ Aggressive/destructive behavior subscale } \\
\hline 1. Hitting & $0.793^{* *}$ & 0.104 \\
\hline 2. Kicking & $0.775^{* *}$ & $0.982^{* *}$ \\
\hline 3. Pushing & $0.691^{* *}$ & $0.940 * *$ \\
\hline 4. Biting & $0.743^{* *}$ & 0.247 \\
\hline 5. Grabbing, pulling & $0.643^{* *}$ & $0.588^{*}$ \\
\hline 6. Scratching & $0.551^{* *}$ & $0.739^{*}$ \\
\hline 7. Pinching & $0.646^{* *}$ & $0.987^{* *}$ \\
\hline 8. Spitting & $0.385^{* *}$ & - \\
\hline 9. Verbally abusive & $0.262^{* *}$ & - \\
\hline 10. Destroys things & $0.516^{* *}$ & 0.443 \\
\hline 11. Cruel act & $0.561^{* *}$ & $0.943^{* *}$ \\
\hline 12. Other & 0.033 & -0.071 \\
\hline
\end{tabular}

${ }^{*} \mathrm{p}<0.05,{ }^{* *} \mathrm{p}<0.01$ 
$0=$ no visible signs of behavior or symptom, $1=$ mild, presence of a problematic behavior or symptom is questionable or mild, $2=$ moderate, clear presence of a problematic behavior or symptom and the severity of the resulting consequence is moderate (e.g., biting self and/or others leaves a teeth mark; the behavior or symptom causes clear interference in activity of the self, others or group), $3=$ severe, clear presence of a severe problematic behavioral or symptom and the severity of the resulting consequence is severe (e.g., biting of self and/or others leaves a marked and long-lasting teeth mark; the behavior or symptom causes severe or long-lasting interference in activity of the self, others or group), and 4=very severe, clear presence of a very severe problematic behavioral or symptom and the severity of the resulting consequence is very severe (e.g., biting of self and/or others results in skin damage or bleeding; the behavior or symptom makes the activity of the self, others or group impossible to initiate or carry on).

\section{Reliability and validity}

\section{Inter-rater reliability}

The inter-rater reliability between 5 independent raters (intra-class correlation) was examined and the ratings were highly correlated: self-harm behavior subscale, $r=0.992(95 \%$ $\mathrm{CI}=0.984-0.997)$, stereotyped behavior subscale, $\mathrm{r}=0.988$ (95\% $\mathrm{CI}=0.978-0.994)$, and aggressive/destructive behavior subscale, $\mathrm{r}=1.000$.

\section{Item-total correlations}

In the self-harm subscale, all items except 5, 8, and 11 showed significant correlations. As for the stereotyped subscale, all items except 21 showed significant correlations. Likewise, all items of the aggressive/destructive subscale showed signifi- cant item-total correlations except for 12 'other' item (Table 2).

\section{Test-retest reliability}

Fifteen participants were re-examined after 2 weeks for test-retest reliability. First, the test-retest correlations for the self-harm behaviors subscale was relatively low $(r=0.233)$ which was attributable to items such as 4 and 14 that showed insignificant level of correlations. Otherwise, high levels of correlations (ranging from $\mathrm{r}=0.629$ to $\mathrm{r}=1.00$ ) were shown in most other items. In terms of the stereotyped behaviors subscale, the test-retest correlation was $\mathrm{r}=0.625$ with items 1,3 , $8,11,14$, and 16 showing high significant correlations (ranging from $r=0.560$ to $r=1.00$ ) while the items $4,13,15,20,21$, 23,24 did not reach the level of significance. Lastly, the aggressive/destructive behaviors subscale showed test-test reliability of $r=0.907$, with items $2,3,5,6,7$, and 11 showing strong significant correlations (ranging from $r=0.739$ to $r=0.987$ ) and other items failing to reach significance (Table 2).

\section{Validity}

As the results of the correlation analysis between the BPI01 subscales and K-SIB-R items, the BPI-01 self-harm behavior subscale total score was found to be significantly positively correlated with self-harm, destructiveness, stereotyped behavior, aggression, withdrawal items of the K-SIB-R. The BPI-01 stereotyped behavior subscale was significantly associated with destructiveness, stereotyped behavior, withdrawal and uncooperativeness items of the K-SIB-R. Lastly, BPI-01 aggressive behavior subscale was significantly correlated with self-harm, other-harm, destructiveness, obstructiveness, aggressiveness, and uncooperativeness items (Table 3).

In terms of the correlations of BPI-01 subscales with KCBLC subscales, only the BPI-01 self-harm subscale was sig-

Table 3. Spearman correlation coefficients between BPI-01 and KSIB-R scales

\begin{tabular}{lllllllll}
\hline \multicolumn{1}{c}{ K-SIB-R } & \multicolumn{1}{c}{1} & \multicolumn{1}{c}{2} & \multicolumn{1}{c}{3} & \multicolumn{1}{c}{4} & \multicolumn{1}{c}{5} & 6 & 7 & 8 \\
\hline BPI-01 & & & & & & & & \\
Self-injury behavior & $0.636^{* * *}$ & 0.197 & $0.340^{* *}$ & 0.166 & $0.375^{* * *}$ & $0.206^{*}$ & $0.259^{*}$ & 0.159 \\
Stereotyped behavior & 0.185 & 0.155 & $0.296^{*}$ & 0.133 & $0.357^{* * *}$ & 0.185 & $0.340^{* *}$ & $0.228^{*}$ \\
Aggression/destruction & $0.230^{*}$ & $0.667^{* * *}$ & $0.461^{* * *}$ & $0.503^{* * *}$ & 0.108 & $0.672^{* * *}$ & 0.188 & $0.429^{* * *}$ \\
\hline
\end{tabular}

${ }^{*} p<0.05,{ }^{* *} p<0.01,{ }^{* * *} p<0.001$. 1: hurtful to self, 2: hurtful to others, 3: destruction of property, 4: disruptive behavior, 5: stereotyped habits, 6: social aggressiveness, 7: social withdrawal, 8: uncooperativeness. BPI-01: Behavior Problems Inventory-01, K-SIBR: Korean-Scales of Independent Behavior-Revised

Table 4. Spearman correlation coefficients between BPI-01 subscales and CBCL symptom-based scales

\begin{tabular}{|c|c|c|c|c|c|c|c|c|c|c|c|c|}
\hline $\mathrm{CBCL}$ & 1 & 2 & 3 & 4 & 5 & 6 & 7 & 8 & 9 & 10 & 11 & 12 \\
\hline \multicolumn{13}{|l|}{ BPI-01 } \\
\hline Self-injury beh & -0.130 & -0.090 & -0.188 & -0.104 & -0.047 & -0.120 & -0.076 & -0.029 & -0.175 & $-0.229 *$ & $-0.208^{*}$ & -0.098 \\
\hline Stereotyped b & 0.075 & 0.120 & -0.027 & 0.075 & 0.142 & 0.094 & 0.131 & 0.080 & -0.038 & -0.050 & -0.033 & 0.090 \\
\hline Aggression/destruction & -0.015 & 0.032 & -0.058 & 0.042 & 0.011 & 0.030 & -0.066 & 0.031 & 0.085 & -0.057 & -0.012 & 0.017 \\
\hline
\end{tabular}

${ }^{*} \mathrm{p}<0.05$. 1: total behavior problem, 2: internalizing problems, 3: externalizing problems, 4: anxiety/depression, 5: withdrawal, 6: somatization, 7: social problems, 8: thought problems, 9: attention problems, 10: delinquent behavior, 11: aggressive behavior, 12: other problems. BPI-01: Behavior Problems Inventory-01, CBCL: Child Behavior Checklist 
nificantly negatively correlated with K-CBCL rule-violation and aggressive behaviors (Table 4). When the individual items of the self-harm subscale were examined, only 'bodyhitting,' 'self-scratching,' 'vomiting, 'self-pinching,' and 'pica' items were significant correlated with some of K-CBCL subscales, especially 'pica' showing significant negative correlations with K-CBCL problematic behavior total score, as well as with externalization, anxiety/depression, somatic symptoms, social immaturity, thought disorder, attentional problems, and aggressive behavior subscales. Among the items of the BPI-01 stereotyped behavior subscales, only items 'sniffing own body,' 'spinning objects,' 'engaging in complex hand and finger movement,' 'maintaining bizarre body postures,' and 'waving hands' showed some significant correlations with the K-CBCL subscales. Lastly, for the BPI-01 aggressive/destructive behavior subscale items, only 'biting,' 'verbally abusive,' 'destroys things,' and 'other' showed a few significant correlations with K-CBCL subscales.

\section{DISCUSSION}

In Korea, the clinical utility of the assessment tools for the behavioral problems in patients with IDD and ASD has been limited due to inadequate standardized translation process, copyright issues, and consideration for cultural and linguistic differences. Also, unlike diagnostic tools, behavioral assessment needs to focus on behavioral issues. In our study, we refined the Korean translation of the BPI-01 to be more appropriate for clinical application, and we confirmed BPI01 showed good psychometric properties with high reliability (internal consistency, inter-rater reliability, and test-retest reliability) and sufficient convergent validity according to interviews with caregivers and observation of behavior by researchers. Our overall results demonstrated the efficacy of the scale to measure a wide range of problematic behaviors in patients with ASD and neurodevelopmental disorders for clinical and research purposes.

In terms of item-total reliability, all items except the "maintaining bizarre body postures” item (Cronbach's alpha=0.197) among 25 items of stereotyped subscale showed significant correlation with the total score of BPI-01, and all items of the aggressive/destructive subscale showed significant itemtotal correlations except for 'other' item. In the case of the self-harming subscale, all items excluding 'vomiting' (Cronbach's alpha=0.16), 'stuffing objects' (Cronbach's alpha=0.19), and 'aerophagia' (Cronbach's alpha $=0.10$ ) showed a statistically significant correlation. In the original development of the BPI scale, item-total correlation of "maintaining bizarre body postures" in the stereotyped behavioral subscale was low (Cronbach's alpha=0.26), and item-total correlations for 'vomiting,' 'stuffing objects,' and 'aerophagia' items were $0.31,0.14$, and 0.19 , respectively. These values were lower than the average, and the results were consistent with this study. ${ }^{12)}$ There is a possibility of low reliability due to small sample size. Previous studies on the reliability and validity of BPI01 for children and adolescents with intellectual disabilities in Asia have shown internal reliability to be high. ${ }^{17)}$

The BPI-01 demonstrated excellent inter-rater correlations across subscales but 2 -week test-retest reliability varied to a large degree among subscales. The non-significant test-retest correlation of the self-injury behavior subscale total score may be partly attributed to the lack of occurrences of some items (e.g., 'drinking') as well as intervention by the staff to prevent or stop the recurrence of these behaviors (e.g., 'selfscratching'). In contrast, the stereotyped behavior subscale total showed a moderate level of test-retest reliability with items 'rocking back and forth,' 'spinning own body,' 'pacing,' 'yelling and screaming,' 'spinning objects' and 'engaging in complex hand and finger movement' showing very strong significant test-retest correlations and aggressive/destructive behavior subscale total score and items 'kicking, 'pushing,' 'grabbing/pulling,' 'scratching,' 'pinching' and 'cruel act' also showed strong test-retest correlations. As with the self-harm behavior subscale, the lack of significant test-retest correlations in some items of the stereotyped behavior subscale and the aggressive/destructive behavior subscale could be attributed to insufficient number of occurrences or cases of such behaviors in our sample. Hence, while the total scores and most items of these scales can be considered to show sufficient testretest reliability, the items lacking significant correlations should be examined further with a larger number of cases.

A moderate concurrent validity of the BPI-01 subscales was obtained using the K-SIB-R items, but there were many overlapping correlations. For example, destructive behavior item of the K-SIB-R was significantly correlated with all BPI-01 subscales, while self-harm item of the K-SIB-R was correlated with only the BPI-01 self-harm behavior and destructiveness behavior subscales, as did stereotyped behavior item of the K-SIB-R with stereotyped behavior and self-harm subscales. Such a pattern of correlations demonstrates the complex nature of the IDD and ASD symptomatology which may be characterized by a constellation of problematic behaviors.

In our study, we observed that the BPI- 01 and K-CBCL were not significantly correlated for the most part, except between the BPI-01 self-harm behavior subscale and the K-CBCL rule violation and aggressive behaviors subscales. On the other hand, a recent study has reported the correlations between the BPI-01 and the K-CBCL subscales to be low to moderate. ${ }^{18)}$ The differences in the results can be attributed to a number of factors. First, there may be differences in the types of 
symptoms to which each scale might be sensitive. Our sample included a relatively high proportion of patients with severe symptoms who were receiving psychiatric drug treatment, hence the overall lack of correlations between the measures may reflect the modest overlap in the range and severity of problem behaviors they cover. Second, the K-CBCL is usually carried out for children of age 6 to 18 but in our study we included those above the age of 18 whose symptoms may have been under-rated because of the different life routine in the daytime. Lastly, the majority of our sample consisted of IDD and ASD, which was quite different from the previous study based predominantly on IDD patients. ${ }^{18)}$

Compared to presently available instruments, the BPI-01 has a distinct advantage of being shorter but comprehensive enough to sufficiently address the characteristic problematic behaviors of IDD and ASD children. Also, the BPI-01 is approved by the original author to be made available for noncommercial use unlike the K-SIB-R and K-CBCL. Hence, the BPI-01 may be able to serve as a valid and reliable alternative for research and clinical assessments.

As the limitation of our study, we included many adult participants in our sample so that the mean age of our participants was above 20 years. In many cases, patients are often referred to the residential caring facilities when repeated problematic behaviors present significant social impairment. The BPI-01 can serve as a useful tool for identifying and preparing for possible problematic behaviors for the caretakers during the referral process. Likewise, in case of the IDD and ASD children being raised at home, the BPI- 01 can be applied to facilitate the identification and assessment of the range of problematic behaviors in hospital or clinical settings, but further study is warranted to standardize the BPI-01 according to the specific age.

In this study, it is possible to evaluate self-harming behavior, stereotyped behavior, and aggressive/destructive behavior based on BPI-01 and to determine the effects of pharmacological and behavior treatments according to severity and frequency of problematic behavior. This is expected to improve the quality of life of patients with developmental disabilities and caregivers.

\section{CONCLUSION}

This study examined the validity and reliability of the Korean version of the BPI- 01 which was translated and modified through the standard translation procedure. The BPI-01 provided a reliable and highly valid assessment of problematic behaviors in IDD and ASD patients and should serve as a helpful tool for the accurate determination of the treatment intervention effects.

\section{Acknowledgments}

This study was supported by a research fund from Seoul National Hospital (2013-10), Ministry of Health \& Welfare, Republic of Korea.

\section{Conflicts of Interest}

The authors have no financial conflicts of interest.

\section{REFERENCES}

1) McKenzie K, Milton M, Smith G, Ouellette-Kuntz H. Systematic review of the prevalence and incidence of intellectual disabilities: current trends and issues. Curr Dev Disord Rep 2016;3:104-115.

2) Kim YS, Leventhal BL, Koh YJ, Fombonne E, Laska E, Lim EC, et al. Prevalence of autism spectrum disorders in a total population sample. Am J Psychiatry 2011;168:904-912.

3) Pantelis PC, Kennedy DP. Estimation of the prevalence of autism spectrum disorder in South Korea, revisited. Autism 2016;20:517-527.

4) American Psychiatry Association. Diagnostic and Statistical Manual of Mental Disorders. 5th ed. Washington, DC: American Psychiatric Publishing;2013.

5) Hong K. Korean textbook of child psychiatry. Seoul: Hakjisa;2014.

6) Intagliata J, Willer B. Reinstitutionalization of mentally retarded persons successfully placed into family-care and group homes. Am J Ment Defic 1982;87:34-39.

7) McGuire K, Fung LK, Hagopian L, Vasa RA, Mahajan R, Bernal P, et al. Irritability and problem behavior in autism spectrum disorder: a practice pathway for pediatric primary care. Pediatrics 2016; 137 Suppl 2:S136-S148.

8) Marcus RN, Owen R, Kamen L, Manos G, McQuade RD, Carson WH, et al. A placebo-controlled, fixed-dose study of aripiprazole in children and adolescents with irritability associated with autistic disorder. J Am Acad Child Adolesc Psychiatry 2009;48:1110-1119.

9) Ji N, Findling RL. An update on pharmacotherapy for autism spectrum disorder in children and adolescents. Curr Opin Psychiatry 2015;28:91-101.

10) Paik EH, Lee BI, Cho SJ. Korean Scales of Independent BehaviorRevised (K-SIB-R). Seoul: Hakjisa;2007.

11) Brown RT, Amler RW, Freeman WS, Perrin JM, Stein MT, Feldman HM, et al. Treatment of attention-deficit/hyperactivity disorder: overview of the evidence. Pediatrics 2005;115:e749-e757.

12) Rojahn J, Matson JL, Lott D, Esbensen AJ, Smalls Y. The Behavior Problems Inventory: an instrument for the assessment of self-injury, stereotyped behavior, and aggression/destruction in individuals with developmental disabilities. J Autism Dev Disord 2001;31:577-588.

13) Aman MG, Singh NN, Stewart AW, Field CJ. The aberrant behavior checklist: a behavior rating scale for the assessment of treatment effects. Am J Ment Defic 1985;89:485-491.

14) Wild D, Grove A, Martin M, Eremenco S, McElroy S, Verjee-Lorenz A, et al. Principles of good practice for the translation and cultural adaptation process for patient-reported outcomes (PRO) measures: report of the ISPOR task force for translation and cultural adaptation. Value Health 2005;8:94-104.

15) Achenbach TM, Edelbrock CS. Manual for the Child Behavior Checklist and Revised Child Behavior Profile. Burlington, VT: Dept. Psychiatry, University of Vermont;1983.

16) Jeong B, Yoo E, Jung M, Kang D, Park S, Park SH. Validity and reliability of the Korean version of the behaviour problems inventory. J Appl Res Intellect Disabil 2013;26:578-590.

17) An X, Rojahn J, Curby TW, Ding Y. Psychometric properties of the Chinese Behavior Problems Inventory-01 in children and adolescents with or at risk for intellectual disabilities. Res Dev Disabil 2014;36C:256-263.

18) Baraldi Gda S, Rojahn J, Seabra AG, Carreiro LR, Teixeira MC. Translation, adaptation, and preliminary validation of the Brazilian version of the Behavior Problems Inventory (BPI-01). Trends Psychiatry Psychother 2013;35:198-211. 